\title{
Changes in resuscitation practice at birth
}

\section{ACL Allwood, RJ Madar, JH Baumer, L Readdy, D Wright}

Arch Dis Child Fetal Neonatal Ed 2003;88:F375-F379

\begin{abstract}
Aim: To investigate secular changes in neonatal resuscitation at birth.
Methods: Single centre observational study of 17890 infants born between May 1993 and April 1997. T-piece ventilation was introduced in April 1995.

Observations: Rates and modes of ventilatory resuscitation, early neonatal encephalopathy, neonatal convulsions, and meconium aspiration syndrome; 1 and 5 min Apgar scores; maternal age and method of delivery; paediatric attendance at delivery and resuscitation. Results: The rate of all forms of ventilatory resuscitation fell during the four year period from $11.0 \%$ to $8.9 \%$. The rate of intubation fell from $2.4 \%$ to $1.2 \%$. A reduced rate of intubation was seen at all gestations of 30 weeks and above. There was no difference in rates of relevant neonatal problems during the period except for a reduction in neonatal convulsions. The introduction of T-piece ventilation did not contribute to the reduction in intubation in a logistic regression model that included time trend.

Conclusion: A marked reduction in the rate of intubation was observed, without any reduction in the efficacy of resuscitation. This may reflect improvements and changing emphasis in resuscitation train-
\end{abstract}

See end of article for authors' affiliations ................... ing.

For correspondence: Dr Allwood, Neonata Intensive Care Unit, Derriford Hospital, Plymouth, Devon
PL6 8DH, UK; alex.allwood@virgin.net

T he provision of adequate ventilation is the cornerstone of resuscitation at birth. The means by which this is best achieved remains contentious. Endotracheal intubation has long been advocated as the definitive method. ${ }^{1}$ It has the advantage of providing a secure airway but requires appropriate training and supervised experience. ${ }^{2}$ The skill is not easily taught or practiced. Significant complications may arise from intubation. Minor localised trauma to the oropharynx is well described. ${ }^{3}$ More serious injuries including vocal cord avulsion and tracheal or oesophageal perforation have been reported ${ }^{4-6}$ Systemic adverse effects of airway manipulation during laryngoscopy and intubation include tachycardia or bradycardia, hypoxaemia, hypertension, ${ }^{7-9}$ and raised intracranial pressure. ${ }^{10-12}$ Such adverse responses have been implicated as contributory factors in the aetiology of intraventricular haemorrhage. ${ }^{13}{ }^{14}$ For these reasons non-invasive ventilatory resuscitation using a mask and self inflating bag valve device or T-piece continues to be the firstline means of newborn ventilatory resuscitation in the United Kingdom.

T-piece ventilation for neonatal resuscitation at birth was described as early as $1913 .{ }^{15}$ Despite further publications in the $1920 \mathrm{~s}^{16}$ and $1960 \mathrm{~s},{ }^{17}$ this method of newborn resuscitation only gained wider acceptance in the late $1980 \mathrm{~s} .{ }^{18}$ The T-piece connects to a facemask to deliver a flow regulated, pressure limited, oxygen supply to the baby, enabling application of prolonged initial inflation breaths. Inflation for at least $2 \mathrm{~s}$ has previously been demonstrated to enhance the establishment of functional residual capacity at birth in intubated babies. ${ }^{19} \mathrm{~A}$ physiological study comparing T-piece ventilation with the best bag valve mask systems demonstrated at least similar efficacy. ${ }^{20}$ A recent study investigated interoperator performance comparing manual bagging devices with T-piece for ventilation of neonatal manikins. ${ }^{21}$ T-piece was found to more consistently deliver target pressures and prolonged inflation independent of the operator's expertise. A previous review of resuscitation practice at birth ${ }^{22}$ cited local clinical experience suggesting that the technique might markedly reduce the need for endotracheal intubation. This has not been formally investigated in any subsequent studies.

Recent data from Canada $^{23}$ investigating adherence to standardised neonatal resuscitation training (Neonatal Resuscitation Program ${ }^{24}$ ) revealed that bag and mask ventilation

was performed in $10.6 \%$ of deliveries and that intubation was undertaken in $3.6 \%$. Previous population based studies from Scandinavia ${ }^{25}$ suggest that unanticipated need for intubation of low risk newborns (defined as those delivered beyond 32 weeks gestation by non-instrumental vertex vaginal delivery without additional staff in attendance prior to birth) is a rare event ( 1 in 5000 deliveries) and that bag and mask ventilation is adequate in the vast majority of cases. There is no comparable United Kingdom data and little information exists regarding changing trends in resuscitation practice. As part of an ongoing system of monitoring, information about neonatal resuscitation was collected prospectively onto computer for all hospital deliveries during a four year period in which T-piece ventilation was introduced. This provided an opportunity to investigate the associated changes in intubation practice in a large population.

\section{POPULATION AND METHODS}

Birth details of all babies born at Derriford Hospital between May 1993 and April 1997 were entered directly into a computerised database within 24 hours of delivery, irrespective of whether the baby was admitted to the neonatal intensive care unit. This included details of maternal age, gestation, birth weight, mode of delivery, 1 and 5 min Apgar scores, presence of medical staff at delivery and/or resuscitation, method of resuscitation and complications (if any), and whether the infant was admitted to the neonatal unit. Normal vaginal delivery was defined as unassisted vertex vaginal delivery through clear liquor at any gestation. The best estimate of gestation was either calculated by the computer when the expected date of delivery was known, or was entered directly. Medical information on neonatal diagnoses, including convulsions in the neonatal period, moderate or severe early neonatal encephalopathy (defined as a clinical course consistent with grade 2 or 3 hypoxic ischaemic encephalopathy ${ }^{26}$ in which no other cause could be attributed), and meconium aspiration syndrome (defined as admission to the neonatal unit following delivery through meconium stained liquor with respiratory symptoms and consistent chest $x$ ray appearances), was recorded on all babies. Delivery and diagnostic information was corroborated by medical staff following consultant led 
Table 1 Population characteristics during the period under investigation. All figures are means (SD) or percentages

\begin{tabular}{lllll} 
& $\begin{array}{l}\text { Year 1 } \\
n=4471\end{array}$ & $\begin{array}{l}\text { Year 2 } \\
n=4415\end{array}$ & $\begin{array}{l}\text { Year 3 } \\
n=4402\end{array}$ & $\begin{array}{l}\text { Year 4 } \\
n=4602\end{array}$ \\
\hline Maternal age* & $27.29(5.32)$ & $27.34(5.38)$ & $27.62(5.38)$ & $27.81(5.50)$ \\
Gestation & $39.33(2.20)$ & $39.34(2.29)$ & $39.36(2.01)$ & $39.34(2.03)$ \\
Birth weight & $3303(598)$ & $3321(606)$ & $3330(591)$ & $3323(580)$ \\
Presence of doctor at delivery* & $35.9 \%$ & $32.0 \%$ & $24.5 \%$ & $22.5 \%$ \\
Doctor attended after delivery & $4.2 \%$ & $5.9 \%$ & $5.9 \%$ & $5.3 \%$ \\
Non-rotational forceps* & $6.2 \%$ & $4.7 \%$ & $3.9 \%$ & $3.8 \%$ \\
Kielland's forceps $\dagger$ & $0.40 \%$ & $0.43 \%$ & $0.23 \%$ & $0.17 \%$ \\
Ventouse extraction* & $2.7 \%$ & $3.1 \%$ & $6.0 \%$ & $5.4 \%$ \\
Breech \pm forceps & $0.78 \%$ & $1.27 \%$ & $0.86 \%$ & $0.89 \%$ \\
Elective caesarean* & $6.6 \%$ & $6.6 \%$ & $5.7 \%$ & $4.6 \%$ \\
Emergency caesarean* & $10.3 \%$ & $9.3 \%$ & $8.8 \%$ & $7.3 \%$ \\
Meconium vaginal delivery* & $3.0 \%$ & $5.6 \%$ & $6.0 \%$ & $6.3 \%$ \\
1 min Apgar & $8.4(1.4)$ & $8.6(1.2)$ & $8.5(1.3)$ & $8.4(1.3)$ \\
Moderate or severe early neonatal encephalopathy & $7(0.16 \%)$ & $1(0.02 \%)$ & $4(0.09 \%)$ & $6(0.13 \%)$ \\
Convulsions* & $22(0.49 \%)$ & $15(0.34 \%)$ & $11(0.25 \%)$ & $5(0.11 \%)$ \\
Meconium aspiration syndrome & $5(0.11 \%)$ & $8(0.18 \%)$ & $4(0.09 \%)$ & $10(0.22 \%)$ \\
\hline * p<0.001, †p<0.02. & & & &
\end{tabular}

weekly data reviews. Caesarean sections were recorded as "emergency" if they were performed for neonatal or maternal reasons necessitating urgent operative delivery (not including unscheduled caesarean sections performed for elective reasons). Missing data were identified by systematic enquiry and subsequently entered from the case notes.

Throughout the study period all newly appointed neonatal senior house officers received an hour's induction in neonatal resuscitation from either a consultant or registrar. The resident registrar would be called to attend all the deliveries attended by new senior house officers until they were judged to be competent in the techniques of neonatal ventilatory resuscitation. Neonatal nurse practitioners were not employed in the maternity unit during the study period.

In April 1995, T-piece circuits and facemasks were introduced onto all the resuscitaires in the maternity unit. Practical training in their use was provided to all medical and midwifery staff. Six months later, the $500 \mathrm{ml}$ self inflating bags and masks that had until then been available were removed from all resuscitaires.

For this study, the computer database was checked against the labour ward register of births and the neonatal intensive care unit manual admission record. Entries in the database relating to outborn babies were excluded from the analysis. Comparison between the labour ward register and the computer database of deliveries on five randomly selected dates during the study period revealed complete case acquisition $(n=56)$. Comparison with the neonatal intensive care unit manual admission record revealed six cases missing from the computer database that were subsequently entered following case note review. Further systematic database interrogation was undertaken to identify missing, anomalous, and outlying data, which were then manually retrieved from case notes or the labour ward register if notes were unobtainable. A total of 184 cases with incomplete or questionable database entries were manually verified. Upon completion of this process there were seven missing items of data pertaining to five cases (two pairs of Apgar scores and three gestations-all concealed pregnancies) yielding data ascertainment in excess of $99.9 \%$. A case note review of a random sample of 20 infants was undertaken. This confirmed completely accurate recording of all variables except gestation, which varied from the gestation written in the case notes in 12. The difference was less than 14 days in all but one case.

\section{STATISTICAL ANALYSIS}

Population characteristics of each of the periods examined were analysed using the student $t$ test for normally distributed data. Unadjusted non-parametric data were compared using the $\chi^{2}$ test. Logistic regression analysis of the two epochs before and after the introduction of T-piece ventilation was undertaken to analyse possible effects of T-piece use on intubation practice, while allowing for the influence of confounding variables. Statistical models were developed using logistic regression to investigate the association of these factors with endotracheal intubation. The analysis incorporated a time trend model enabling the independent effect of temporal changes in resuscitation practice to be investigated.

In addition to investigation of factors associated with newborn intubation by use of logistic regression, crude intubation rates for the lowest risk deliveries were derived, enabling direct comparison of United Kingdom practice with previously published data on the Scandinavian experience in this subgroup. ${ }^{25}$

\section{RESULTS}

Complete data were available for 17885 babies born between May 1993 and April 1997. There was a significant increase in mean maternal age (by 0.52 years) from the first to the last of the 4 years in the study period (table 1). Rates of forceps deliveries and elective and emergency caesarean sections fell significantly, while increases in the rate of ventouse extractions and vaginal deliveries through meconium were observed. Progressively fewer deliveries were electively attended by medical staff during the 4 years studied but there was no significant change in the number of babies attended by a paediatrician after delivery. The rates of early neonatal encephalopathy and meconium aspiration syndrome did not change significantly during the study period. However, the rate of neonatal convulsions fell by $78 \%$.

There was a statistically significant temporal reduction in rate of intubation throughout the study period $(\mathrm{p}=0.016$, odds ratio 0.93 for each successive quarter year from the start of the study period). The crude rate of endotracheal intubation (all gestations and modes of delivery) decreased from $2.4 \%$ in the first year to $1.2 \%$ in the final year, a $51 \%$ reduction $\left(\chi^{2}\right.$ test for trend 29.2, p<0.001). The frequency of ventilatory resuscitation using any method likewise fell from $11.0 \%$ to $8.9 \%\left(\chi^{2}\right.$ test for trend $14.4, \mathrm{p}<0.001)$. The proportion of babies receiving ventilatory support at birth who were managed without endotracheal intubation increased from $76 \%$ to $86 \%\left(\chi^{2}\right.$ test for trend 19.8, $\mathrm{p}<0.001)$. Analysis by gestational subgroups is shown in table 2 . There was a significant reduction in rates of intubation of babies of 30 weeks' gestation and above. Of the 12473 babies born by normal vaginal delivery throughout the 
Table 2 Intubation rates by gestation

\begin{tabular}{|c|c|c|c|c|c|}
\hline & \multicolumn{4}{|c|}{ Proportion intubated } & \multirow[b]{2}{*}{$\chi^{2}$ Test for trend } \\
\hline & Year 1 & Year 2 & Year 3 & Year 4 & \\
\hline$<30$ weeks & $34 / 40(85.0 \%)$ & $44 / 55(80.0 \%)$ & $22 / 27(81.5 \%)$ & $27 / 33(81.8 \%)$ & 0.05 (NS) \\
\hline 30-34 weeks & $22 / 85(25.9 \%)$ & $19 / 85(22.4 \%)$ & $9 / 83(10.8 \%)$ & $6 / 66(9.1 \%)$ & $11.02(p<0.001)$ \\
\hline 34-37 weeks & $14 / 226(6.2 \%)$ & $4 / 217(1.8 \%)$ & $5 / 227(2.2 \%)$ & $3 / 243(1.2 \%)$ & $7.72(p<0.01)$ \\
\hline 37 weeks + & $34 / 4117(0.83 \%)$ & $28 / 4055(0.69 \%)$ & $19 / 4065(0.47 \%)$ & $17 / 4260(0.40 \%)$ & $8.15(p<0.01)$ \\
\hline
\end{tabular}

Table 3 Logistic regression analysis of factors associated with intubation

\begin{tabular}{llll}
\hline & Significance & \multicolumn{2}{l}{ Odds ratio $(95 \% \mathrm{Cl})$} \\
\hline T-piece era $v$ baseline era & $0.9(\mathrm{NS})$ & $1.03(0.58$ to 1.80$)$ \\
Time trend* & 0.016 & $0.93(0.87$ to 0.99$)$ \\
Increasing gestational age $\dagger$ & $<0.0001$ & $0.66(0.61$ to 0.71$)$ \\
Neville Barnes' forceps $\ddagger$ & $<0.001$ & $4.39(2.06$ to 9.36$)$ \\
Kielland's forceps $\ddagger$ & $<0.01$ & $11.9(2.56$ to 55.67$)$ \\
Ventouse extraction $\ddagger$ & $<0.001$ & $4.49(2.16$ to 9.31$)$ \\
Vaginal breech delivery $\ddagger$ & $<0.0001$ & $9.43(4.85$ to 18.34$)$ \\
Elective caesarean & 0.58 (N.S.) & $1.21(0.61$ to 2.4$)$ \\
Emergency caesarean $\ddagger$ & $<0.0001$ & $7.14(5.05$ to 10.12$)$ \\
Meconium vaginal delivery $\ddagger$ & $<0.0001$ & $5.39(2.69$ to 10.77$)$ \\
\hline
\end{tabular}

*Three month increments from start of study period.

†Effect of one week incremental increase in gestational age

$\ddagger$ Relative to normal vaginal delivery in which crude intubation rate $0.6 \%$.

study period, $3.7 \%$ received mask ventilation and $0.6 \%$ were intubated. In comparison, $8.3 \%$ of babies born by elective caesarean section received mask ventilation ( $\chi^{2}$ test 53.9, $\mathrm{p}<0.001$ ), increasing to $19.1 \%$ of those born by emergency caesarean section $\left(\chi^{2}\right.$ test $\left.1502, \mathrm{p}<0.001\right)$.

Complete information was available for 8494 babies prior to the introduction of T-piece ventilation in April 1995 and for 9391 babies following its introduction. Analysis of factors associated with intubation using a logistic regression model and allowing for the influence of confounding variables revealed a statistically significant $(p=0.016)$ time trend associated with intubation practice. However, T-piece ventilation per se was not independently associated with reduced risk of intubation. The independent association of these and other factors with intubation are shown in table 3. Quoted odds ratios for association of differing modes of delivery with intubation are relative to normal vaginal delivery and independent of gestation.

Of all infants throughout the study period, $2.1 \%$ had a low Apgar score (less than 4 at $1 \mathrm{~min}$ and/or less than 7 at $5 \mathrm{~min}$ ). There was no significant difference between the two groups in the proportion of infants $(31 \%$ and $27 \%$ in the two time periods) with a low (less than 4) 1 min Apgar score whose 5 min Apgar scores remained low.

A paediatrician was present at 5133 of the 17890 deliveries (28.6\%). In 3867 (75\%) of such cases the attendant paediatric staff did no more than provide stimulation, undertake oropharyngeal suction, or administer facial oxygen. Twenty per cent of babies delivered with a paediatrician attending received some form of ventilatory resuscitation but only $3.4 \%$ were intubated. In contrast, 948 babies received medical attention after birth following delivery in the absence of a paediatrician (5.3\% of all deliveries). Four hundred and nineteen $(44 \%)$ of such babies received some form of ventilation and 133 were intubated-14\% of all newborns attended urgently after delivery. Of 11461 low risk deliveries (defined as delivery at 32 weeks or more gestation by non-instrumental vertex vaginal delivery without paediatric staff present) a paediatrician attended after birth on 477 occasions (4.2\%).
Two hundred and seventy five $(57.6 \%)$ of these babies received mask ventilation (bag valve mask or T-piece) $-2.5 \%$ of the low risk group overall. Nine babies were intubated-1 in 1273 of the low risk group.

\section{DISCUSSION}

This study demonstrates a marked and significant fall in the incidence of endotracheal intubation at birth in infants above 30 weeks' gestation occurring progressively over a period during which T-piece ventilation replaced bag valve mask resuscitation. The time trend used in the logistic regression model can be considered to be a proxy for factors not individually included in the analysis that may have influenced intubation practice during the study period. The reasons for the reduced intubation rate are unclear, but may include changing obstetric practice or changes in education of paediatric and/or midwifery staff.

Obstetric practice did change during the study period as evidenced by falling rates of caesarean section and forceps deliveries and increasing rates of ventouse extractions. Although the reduction in neonatal convulsions may have reflected improvements in obstetric care, there was no change in 1 min Apgar score during the study period to explain the reduction in ventilatory resuscitation. The rate of early neonatal encephalopathy did not change during the study period. The logistic regression model used to investigate associations with the introduction of T-piece ventilation allowed adjustment for confounding factors (such as mode of delivery) that had changed, although some important influences, such as intrapartum foetal monitoring data, were not available to be incorporated into this study.

This study also provides evidence for which deliveries are most likely to require the presence of a professional capable of undertaking intubation. Apart from prematurity, the highest relative risk was seen in Kielland's rotational forceps and in breech deliveries. Delivery by elective caesarean section appeared to confer an increased risk of mask ventilatory resuscitation (but not intubation) compared with normal vaginal delivery, irrespective of gestation. Emergency caesarean section is significantly associated with increased relative risk of both mask ventilation and intubation. In contrast, babies delivered beyond 32 weeks' gestation by noninstrumental vertex vaginal delivery without paediatric staff being summoned prior to birth are extremely unlikely to require endotracheal intubation unexpectedly at birth. The rarity of such an occurrence supports the emphasis of current newborn life support training ${ }^{27}$ towards adequate airway control and effective mask ventilation techniques rather than endotracheal intubation. It also suggests that in such circumstances it is not necessary for a professional capable of intubation to attend the delivery.

The lack of association of introduction of T-piece ventilation with falling rate of intubation when time trend was included in the model does not exclude an effect, but suggests that this was not the main reason for the reduction. The associated fall in the rate of all forms of ventilatory resuscitation despite unchanged 1 min Apgar scores suggests that improved 
management of resuscitation by both midwifery and paediatric staff may have contributed. It is likely that better recognition of babies not in need of ventilatory support at birth resulted in less unnecessary resuscitation. It is also possible that the observed reduction in intubation rate was part of a continuing trend over a much longer period. It is of note that this has occurred despite reduced experience of neonatal resuscitation by individual doctors in training consequent upon changing patterns of junior doctors' working hours.

There was no deliberate change in resuscitation training or change in policy of grade of medical staff attending deliveries during the study period. However, an audit of local resuscitation practice was presented at the end of the first year of the study period, following which paediatric medical staff were no longer required to electively attend instrumental deliveries at term. Additionally, training in the use of T-piece accompanying its introduction at the end of the second year of the study period may have enhanced awareness of newborn resuscitation and influenced its efficacy. Moreover, the increasing uptake of paediatric life support courses by junior paediatric medical staff during the study period may have improved general awareness of effective life support techniques in the newborn. Specifically, the past decade has seen a change in the emphasis of resuscitation training (at all ages) towards improved airway control and effective mask ventilation rather than mandatory early intubation. Notably, this study preceded the introduction of formal training based upon the Northern Neonatal Network's "Resuscitation at Birth" course, ${ }^{18}$ which has subsequently evolved into the UK Resuscitation Council endorsed Newborn Life Support course. ${ }^{27}$ Despite the availability of such programmes, recent national surveys have demonstrated persisting inadequacies in neonatal resuscitation training of both midwifery staff ${ }^{28}$ and paediatric general professional trainees. ${ }^{29}$

This unit had used $500 \mathrm{ml}$ bag valve mask systems, although $250 \mathrm{ml}$ self inflating bags are not infrequently provided for the purposes of neonatal resuscitation. These smaller bags are reported to achieve tidal volumes rarely exceeding anatomical dead space in babies beyond 35 weeks gestation. ${ }^{30}$ Such devices probably exert any apparent resuscitative effect by virtue of the induction of Head's paradoxical reflex. ${ }^{31}$ It is possible that a reduction in intubation rates would be observed following introduction of the T-piece ventilation system in units currently using low volume self inflating bags.

The rate of low Apgar scores (less than 4 at 1 min and/or less than 7 at $5 \mathrm{~min}$ ) in this population is comparable to previous reports, ${ }^{25}{ }^{32}$ which have demonstrated that up to $80 \%$ of babies requiring ventilatory support at birth can be satisfactorily resuscitated without endotracheal intubation. This study has demonstrated that $86 \%$ of infants receiving T-piece ventilatory support can be managed without intubation in the hands of trained staff. The 5 min Apgar scores of those resuscitated using T-piece ventilation were similar to those seen using $500 \mathrm{ml}$ self inflating bag valve mask, confirming the adequacy of resuscitation using T-piece ventilation.

Preterm babies between 30 and 37 weeks' gestation demonstrated the most marked relative reduction in intubation rates. The reason for this is uncertain, but it is possible that in this gestational subgroup the provision of correctly applied airway management and effective initial inflation breaths may be more critical than in term babies. There was no significant change in the proportion of births between 30 and 37 weeks' gestation attended by a doctor to account for such a marked reduction in intubation rate in this subgroup.

Term babies also demonstrated a significant reduction in intubation rates, although less marked than for preterm babies. This may reflect changes in the management of babies delivered through meconium stained liquor. Current evidence suggests little benefit from intubation and tracheal suctioning of such babies, particularly if vigorous at birth. ${ }^{33}$
There was no reduction in intubation rate in infants less than 30 completed weeks' gestation. It is likely that this reflects the practice of elective intubation for prophylactic surfactant administration in this gestational subgroup.

In conclusion, this study has demonstrated falling intubation rates at birth, particularly marked between 30 and 37 weeks' gestation, during a four year period in a single centre, without accompanying deterioration in 5 min Apgar scores and with reduced rates of neonatal convulsions. The study also provides evidence for the safety and efficacy of T-piece ventilation as an alternative to bag valve mask ventilation in neonatal resuscitation but suggests that other factors, possibly improved education with regard to non-invasive airway support, has contributed more to falling intubation rates.

\section{Authors' affiliations}

ACL Allwood, RJ Madar, JH Baumer, Derriford Hospital, Plymouth, UK L Readdy, D Wright, School of Mathematics \& Statistics, University of Plymouth, UK

\section{REFERENCES}

1 Flagg PJ. The treatment of asphyxia in the newborn. JAMA 1928:91:788-91.

2 Barrie H. Resuscitation of the newborn Lancet 1963:i:650-5.

3 MacPherson TA, Shen-Schwarz S, Valdes-Dapena M. Prevention and reduction of iatrogenic disorders in the newborn. In: Guthrie RD, ed. Clinics in critical care medicine: neonatal intensive care. New York: Churchill Livingstone, 1988;13:271-312.

4 Wohl DL. Traumatic vocal cord avulsion in a newborn. J Voice 1996;10(1):106-8

5 Serlin SP, Dally WJR. Tracheal perforation in the neonate: a complication of endotracheal intubation. J Paediatr 1975;86:596-7.

6 Seefelder C, Elango S, Rosbe KW, et al. Oesophageal perforation presenting as oesophageal atresia in a premature neonate following difficult intubation. Paediatr Anaesth $2001 ; 11(1): 112-18$.

7 Kelly MA, Finer NN. Nasotracheal intubation in neonates: physiological responses and effects of atropine and pancuronium. J Paediatr 1984; 105:303-9.

8 Marshall TA, Deeder R, Pai S, et al. Physiologic changes associated with endotracheal intubation in preterm infants. Crit Care Med 1984;12:501-4.

9 Gibbons PA, Swedlow DB. Changes in oxygen saturation during elective tracheal intubation in infants. Anesth Analg 1987;66:874-8.

10 Raju TNK, Vidyasagar D, Torres C, et al. Intracranial pressure during intubation and anaesthesia in infants. J Paediatr 1980;96:860-2.

11 Stow PJ, McLeod ME, Burrows FA, et al. Anterior fontanelle pressure responses to tracheal intubation in the awake and anaesthetised infant. BrJ Anaesth 1988:60:167-70.

12 Millar C, Bissonnette B. Awake intubation increases intracranial pressure without affecting cerebral blood flow velocity in infants. Can J Anaesth 1994;41:281-7.

13 McDonald MM, Kloops BL, Johnson ML, et al. Timing and antecedents of intracranial haemorrhage in the newborn. Pediatrics 1984;74:32-6.

14 Bada HS, Miller JE, Menke JA, et al. Intracranial pressure and cerebral arterial pulsatile flow measurements in neonatal intraventricular haemorrhage. J Pediatr 1982;100:291-6.

15 Meltzer SJ. Simple devices for effective artificial respiration in emergencies. JAMA 1913:60:1407-10.

16 Henderson $Y$. The prevention and treatment of asphyxia in the newborn. JAMA 1928;90:583-6.

17 Johnson KG, Babson SG. Resuscitation of the apneic premature infant. Pediatrics 1967;40:99-100.

18 Richmond S, ed. Principles of resuscitation at birth. 5th edn. Newcastle-upon-Tyne: Northern Neonatal Network, 1996.

19 Vyas $\mathbf{H}$, Milner AD, Hopkin IE, et al. Physiological responses to prolonged and slow rise inflation. J Pediatr 1981;99:635-9.

20 Hoskyns EW, Milner AD, Hopkins IE. A simple method of face mask resuscitation at birth. Arch Dis Child 1987:62:376-8.

21 Finer NN, Rich W, Craft A, et al. Comparison of methods of bag and mask ventilation for neonatal resuscitation. Resuscitation 2001;49(3):299-305

22 Milner AD. Resuscitation of the newborn. Arch Dis Child 1991;66:66-9.

23 Singhal N, McMillan DD, Yee WH, et al. Evaluation of the effectiveness of the standardized neonatal resuscitation program. J Perinatol $2001 ; 21(6): 388-92$.

24 Neonatal Resuscitation Program. Textbook of neonatal resuscitation. Dallas: American Heart Association \& American Academy of Paediatrics, 2001.

25 Palme-Kilander C. Methods of resuscitation in low-Apgar-score newborn infants - a national survey. Acta Paediatr 1992;81:739-44

26 Sarnat HB, Sarnat MS. Neonatal encephalopathy following foetal distress. A clinical and electroencephalographic study. Arch Neurol 1976;33(10):696-705 
27 Richmond S, ed. Resuscitation at birth - newborn life support provider course manual. London: Resuscitation Council (UK), 2001.

28 Gnanalingham MG, Robinson C, Mir NA. A national review of neonatal resuscitation programmes for midwives [letter]. Arch Dis Child Fetal Neonatal Ed 2001:85:F145

29 Rangaraj S, Rangaraj J, Scholler I, et al. A survey of neonatal resuscitation training provided to general professional trainees at neonatal units in England and Wales [letter]. Arch Dis Child Fetal Neonatal Ed 2001;85:F225.
30 Field D, Milner AD, Hopkin IE. Efficiency of manual resuscitators at birth. Arch Dis Child 1986;61:300-2.

31 Milner AD, Vyas $\mathrm{H}$, Hopkin IE. Efficacy of facemask resuscitation at birth. BM 1984;289:1563-5.

32 Nelson KB, Ellenberg JH. Apgar scores as predictors of chronic neurologic disability. Pediatrics 1981;68(1):36-44.

33 Wiswell TE, Gannon CM, Jacob J, et al. Delivery room management of the apparently vigorous meconium-stained neonate: results of the multicenter international collaborative trial. Pediatrics 2000;105:1-7.

\section{Archimedes}

In order to give the best care to patients and families, paediatricians need to integrate the highest quality scientific evidence with clinical expertise and the opinions of the family. Archimedes is a bimonthly section in Archives which seeks to assist practising clinicians by providing "evidence based" answers to common questions which are not at the forefront of research but are at the core of practice.

The format of Archimedes may be familiar. A description of the clinical setting is followed by a structured clinical question and a brief report of the search. The best evidence available to answer the question is provided as a summary table (which is electronically linked to more detailed appraisals). To pull the information together, a commentary follows. Finally, to make it all much more accessible, a box provides the clinical bottom line.

This month the following topics have been published:

- Is elective high frequency oscillatory ventilation better than conventional mechanical ventilation in very low birthweight infants?

- Do oral antihistamines stop the itch of atopic dermatitis?

- Can surfactant cure babies with severe bronchiolitis?

Previous Archimedes questions can be found in the issues of Archives published in the Fetal and Neonatal edition months since September 2001. Readers wishing to submit their own questions-with best evidence answers-are encouraged to read the Instructions for Authors at http://www.archdischild.com. 